\title{
Waldzustand in der Schweiz: Erfassung, Entwicklung und Einflussfaktoren
}

\author{
Matthias Dobbertin \\ Christian Hug \\ Lorenz Walthert
}

Eidgenössische Forschungsanstalt für Wald, Schnee und Landschaft $(\mathrm{CH})^{*}$ Eidgenössische Forschungsanstalt für Wald, Schnee und Landschaft $(\mathrm{CH})$

Eidgenössische Forschungsanstalt für Wald, Schnee und Landschaft (CH)

\section{Forest condition in Switzerland: assessment, development and influencing factors}

\begin{abstract}
In the early 1980s it was feared that air pollution would cause a widespread forest decline and a reduction in forest productivity. In Switzerland as in most European countries crown defoliation and foliage discoloration were selected as the most important indicators of forest condition. The Sanasilva inventory on a systematic network of plots showed an increase in the proportion of trees with high defoliation until 1995. Since then no trend has been detected. However, large annual fluctuations were often observed following years with large-scale climatic events, such as the storms Vivian and Lothar and the heat summer of 2003. Although highly variable, neither mortality nor removal rates have shown any time trend since 1985. The annual differences in crown defoliation, mortality and tree growth are mainly related to climatic factors. In addition to the climatic conditions, nutrient availability, soil water holding capacity and to a lesser extent air pollution determine the degree of crown defoliation at a given site and for a given species. Although several studies have found a negative effect of nitrogen deposition or ozone concentrations on tree foliation, others have shown that up to now nitrogen deposition has increased tree growth on nitrogen-limited sites. It can thus be concluded that presently air pollution does not pose a direct threat to tree conditions in Switzerland. However, the assessment of crown condition alone is not a suitable tool to detect the effects of air pollution on forests. This requires more detailed measurements on long-term research sites. However, as crown condition is strongly affected by site conditions and climate, it should be further monitored, particularly with regard to the predicted climate change.
\end{abstract}

Keywords: crown defoliation, discoloration, nutrient availability, tree mortality, air pollution doi: $10.3188 /$ szf.2012.0331

* Zürcherstrasse 111, CH-8903 Birmensdorf, E-Mail matthias.dobbertin@wsl.ch

Z u Beginn der 1980er-Jahre bestand in vielen Teilen Europas die Befürchtung, dass die Luftschadstoffe zu einem verbreiteten Waldsterben oder zumindest zu einer deutlichen Verschlechterung des Waldzustandes führen würden und es zu bedeutenden Produktionsrückgängen kommen könnte. Im süddeutschen Raum und in den angrenzenden Regionen starben verbreitet Tannen ab. Meldungen von zusammenbrechenden Fichtenwäldern aus dem schwer durch Luftverschmutzung belasteten Erz- und Riesengebirge erreichten die westliche Öffentlichkeit. In Skandinavien wurden versauernde Seen beobachtet, was zur Beschreibung des «sauren Regens» - verursacht durch im Regenwasser gelöstes Schwefeldioxid $\left(\mathrm{SO}_{2}\right)$ und durch Stickoxide $\left(\mathrm{NO}_{\mathrm{X}}\right)-$ führte. Mit der Veröffentlichung der Ergebnisse einer Untersuchung im Solling in Norddeutschland wurden versauernde Einträge in den Waldboden erstmals als Ursache von Waldschäden in Erwägung gezogen (Ulrich et al 1980).
In der Schweiz beobachteten Forstleute an verschiedenen Orten Schäden an Waldbäumen, und es stellte sich die Frage, ob diese Schäden durch Luftschadstoffe verursacht wurden. Europaweit wurden erste Versuche unternommen, den Waldzustand zu erfassen. In der Schweiz wurde 1985 begonnen, auf einem systematischen Teilnetz des Schweizerischen Landesforstinventars jährlich die Sanasilva-Inventur durchzuführen. Als wichtigstes Merkmal zur Beschreibung des Waldzustands wird seither in der ganzen Schweiz der Anteil Bäume mit starker Kronenverlichtung, das heisst mit einer hohen Abweichung der Belaubung von jener eines optimal belaubten Baumes, erhoben.

In der vorliegenden Arbeit wird untersucht, ob und wie sich der Waldzustand der Schweiz seit 1985 verändert hat und welches die möglichen Ursachen dafür sind. Die Ergebnisse werden mit anderen nationalen und internationalen Untersuchungen verglichen und diskutiert. Abschliessend wird 
eine Bewertung zur Erhebung des Waldzustands mittels Kronenansprachen, insbesondere des Merkmals der Kronenverlichtung, vorgenommen.

\section{Methoden}

\section{Sanasilva-Inventur}

Nach ersten lokalen Erhebungen begann im Jahr 1984 die erste landesweit mit denselben Feldequipen durchgeführte Sanasilva-Inventur. Im ersten Jahr fand sie nur auf Trakten im öffentlichen Wald statt. Seit 1985 wird die Inventur für den gesamten Wald auf einem Teilnetz des Landesforstinventars durchgeführt. In den Jahren 1985 bis 1992 wurde die Inventur auf einem 4×4-km-Netz, in den Jahren 1993, 1994 und 1997 auf einem $8 \times 8$-km-Netz und in den Jahren 1995, 1996 sowie seit 1998 noch auf dem aktuellen 16×16-km-Netz aufgenommen (Abbildung 1). Dabei werden im fünf Aren grossen Probekreis alle Bäume ab $36 \mathrm{~cm}$ Brusthöhendurchmesser (BHD) aufgenommen, im inneren Probekreis von zwei Aren alle Bäume ab $12 \mathrm{~cm}$ BHD. Es werden also auch unterständige Bäume erfasst. Die Auswertung erfolgt stamm-

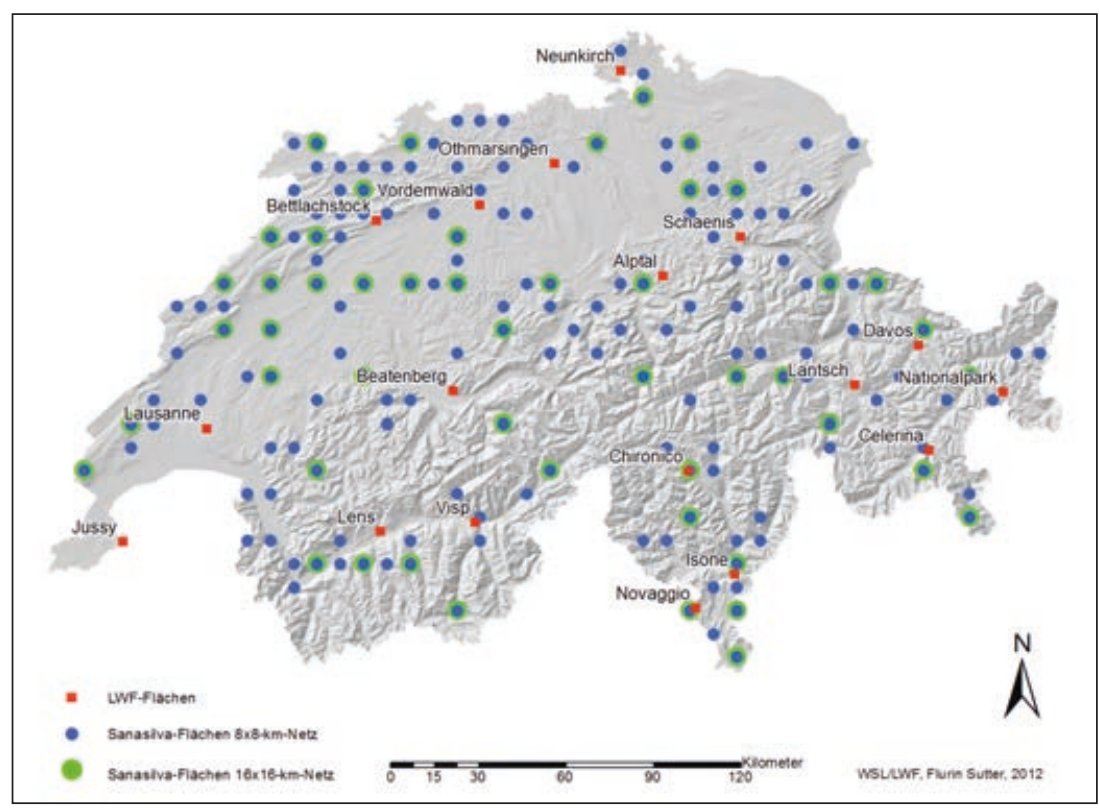

Abb 1 Probeflächen der Sanasilva-Inventur und LWF-Flächen.
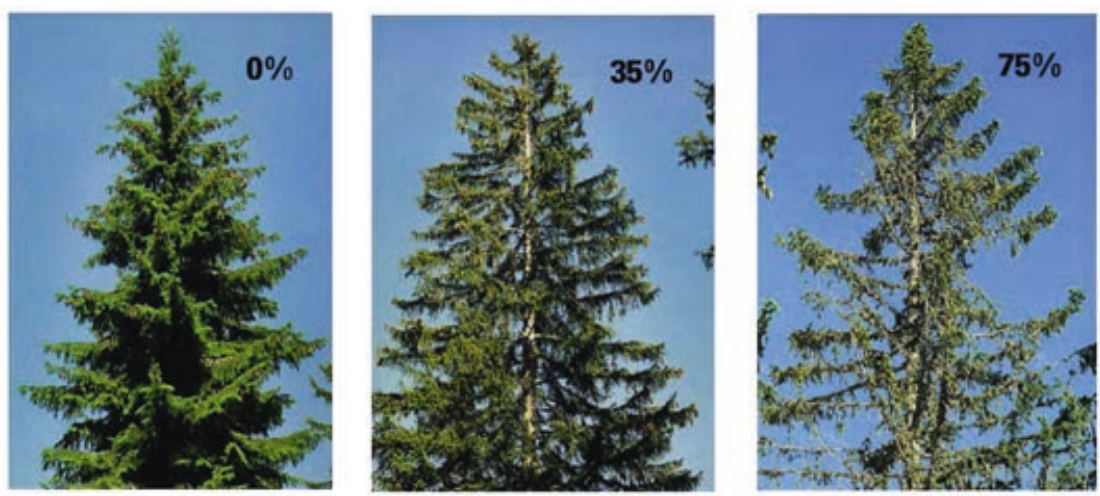

Abb 2 Fichten mit «Bürstenfichten»-Habitus mit unterschiedlicher Kronenverlichtung aus dem Sanasilva-Kronenbilderbuch (Müller \& Stierlin 1990). zahlbasiert. Dabei werden Bäume mit einem BHD von 12 bis $36 \mathrm{~cm}$, welche nur im inneren Probekreis aufgenommen werden, ihrer kleineren Aufnahmefläche entsprechend 2.5-mal höher gewichtet. Darin weicht die Sanasilva-Inventur von den meisten europäischen Erhebungen ab, in welchen nur herrschende und mitherrschende Bäume beurteilt werden.

Die Daten der Kronenansprachen werden im ICP Forests ${ }^{1}$ von fast allen Ländern Europas erhoben und zu Vergleichszwecken zentral in einer Datenbank gespeichert (Level-I-Flächen). Ab 1995 wurden in der Schweiz zusätzlich langfristige Waldökosystemforschungsflächen (LWF-Flächen; entsprechend den Level-II-Flächen des ICP Forests) eingerichtet, auf welchen ebenfalls jährlich eine Kronenzustandserhebung durchgeführt wird (Abbildung 1).

\section{Erhebung der Kronenverlichtung}

Auf der Suche nach einem einfach zu erhebenden Indikator für die Erfassung der langfristigen Entwicklung des Waldzustands entschied man sich für die Erfassung des Nadel- beziehungsweise Blattverlustes (Kronenverlichtung), wobei die aktuelle Belaubung in Prozent einer voll belaubten Baumkrone angegeben wird. Zur Standardisierung und als Referenz wurden für jede Baumart Bilder von Baumkronen mit verschiedener Kronenverlichtung angelegt (Abbildung 2; Müller \& Stierlin 1990). Gleichzeitig wird mithilfe von Trainingskursen versucht, die Schätzung der Feldequipen Jahr für Jahr auf dem gleichem Niveau zu halten. Neben der gesamten Kronenverlichtung (im Folgenden Gesamtverlichtung genannt) wird auch der Anteil der Verlichtung erfasst, der nicht durch bekannte Ursachen wie Insektenfrass oder Frostschaden erklärt werden kann (im Folgenden Kronenverlichtung unbekannter Ursache genannt). Die Gesamtverlichtung wird erst seit 1990 dokumentiert. Die am Einzelbaum erhobenen Daten werden für die Schweiz, für eine Region oder für eine Baumart zusammengefasst. Dabei wird traditionell der Anteil der Bäume mit mehr als 25\% Kronenverlichtung dargestellt, da solche Bäume als geschädigt im Sinne einer deutlichen Abweichung vom Normalzustand angesehen werden. Obwohl diese Grenze heute zunehmend infrage gestellt wird, wird sie für den langfristigen Vergleich beibehalten.

In den Nachbarländern wird die Kronenverlichtung der Bäume meist in vier verschiedenen Klassen dargestellt. Österreich beispielsweise erhob in einer nationalen Zustandserfassung einerseits die Anteile der Bäume in den verschiedenen Verlichtungsklassen und unterschied andererseits flächenbezogene Verlichtungskategorien, um auch die räumliche Verteilung der Kronenverlichtung zu berücksichtigen (Neumann 1989a).

\footnotetext{
1 International Co-operative Programme on Assessment and Monitoring of Air Pollution Effects on Forests
} 


\section{Farbe der Belaubung}

Seit 1993 wird auf den Sansilva-Flächen auch die Farbe der Belaubung der Bäume mittels Standardfarben (Munsell Color Charts für Vegetation) erhoben (Innes et al 1996). Dabei werden mithilfe eines Farbkreises der Farbton, die Reinheit der Farbe und ihre Helligkeit bestimmt. Für die vorliegende Auswertung interessierte nur der Farbton. Zunächst wurde der mittlere flächengewichtete Farbton der vor- und mitherrschenden Bäume für die Hauptbaumarten bestimmt, dann wurde pro Baumart und Fläche der Anteil Bäume ermittelt, deren Farbton deutlich von der mittleren Farbe abwich (für manche Baumarten sind dies zum Beispiel hellgrüne Farbtöne). Zuletzt wurde für jede Probefläche der Anteil Bäume mit mindestens 10\% gelben Nadeln oder Blättern erhoben.

\section{Schadursachen und Samenbehang}

Im Rahmen der Sanasilva-Inventur werden die erkennbaren Schadursachen wie Wind-, Frost- oder Insektenschäden bestimmt. Zudem wird aufgenommen, ob ein Baum lebt oder abgestorben ist, steht oder liegt, genutzt wurde oder sonst wie verschwunden ist. Diese Daten werden genutzt, um Sterbe- und Nutzungsraten zu berechnen und um abzuschätzen, ob gewisse Schadursachen zugenommen haben.

Jeder Baum wird zudem auf den Samenbehang hin beurteilt (keine, wenige oder viele Samen sichtbar). Da dieses Merkmal von 1996 bis 2005 nicht aufgenommen wurde, wird in der vorliegenden Arbeit der Zusammenhang zwischen Samenbehang und Kronenverlichtung für die Jahre 2009 und 2011 untersucht. Diese beiden Jahre eignen sich deshalb besonders gut, weil sie nicht nur ausgeprägte Buchenund Fichtenmastjahre waren, sondern auch auf Jahre folgten, in denen nur wenige Bäume Samen produzierten.

\section{Bodenprofile und Schadstoffeinträge}

Um mögliche Ursachen für die Veränderungen des Waldzustandes in der Schweiz zu bestimmen, wurden auf dem $8 \times 8-\mathrm{km}-\mathrm{Netz}$ der Sanasilva-Inventur in den Jahren 1993 und 1994 rund 170 Bodenprofile gegraben. Die morphologischen, physikalischen und chemischen Bodenuntersuchungen erlaubten es, den Säurezustand ( $\mathrm{pH} \mathrm{CaCl}_{2}$ ) abzuschätzen, die Menge an pflanzenverfügbaren Nährstoffkationen $(\mathrm{Ca}, \mathrm{Mg}$, K) zu bestimmen, das Verhältnis von organischem Kohlenstoff zu Stickstoff (C/N-Verhältnis) für verschiedene Bodentiefen zu ermitteln (Methodenbeschreibung in Walthert et al 2004) sowie Gründigkeit, Wasserspeicherfähigkeit und Wasserhaushalt zu berechnen.

Als Indikatoren für die Luftverschmutzung wurden für die Jahre vor 2000 die N- und S-Einträge (Augustin \& Achermann 2012, dieses Heft) und für das Jahr 1995 die Ozonbelastung (AOT40-Werte;
Braun \& Rihm 2012, dieses Heft) verwendet. Die Jahresmitteltemperaturen und die mittleren Jahresniederschläge von 1961 bis 1990 wurden mit dem Daymet-Programm berechnet (Thornton et al 1997).

\section{Multivariate Auswertung}

Mithilfe einer multivariaten Auswertung wird anhand der drei letzten Sanasilva-Erhebungen auf dem 8×8-km-Netz (1993, 1994 und 1997) untersucht, welche Standortfaktoren und Luftschadstoffe am besten mit der Verlichtung und Verfärbung der Baumkronen korrelieren. Für die Hauptbaumarten Fichte (Picea abies), Buche (Fagus sylvatica) und Tanne (Abies alba) wurden nur lebende, vor- und mitherrschende Bäume ausgewertet.

Als abhängige Variablen wurden pro Stichprobe die mittlere Gesamtverlichtung, die mittlere Kronenverlichtung unbekannter Ursache, der mittlere Farbton der Belaubung, der Anteil Bäume mit Abweichungen vom Normalfarbton und der Anteil Bäume mit ins Gelbe abweichendem Farbton gewählt. Die unabhängigen Variablen wurden in folgende Themen unterteilt:

- Klima: jährlicher Niederschlag, Temperaturen - Wasserverfügbarkeit: Trockenindex der Sommermonate (AT/PT), Wasserspeicherfähigkeit bis $120 \mathrm{~cm}$ Bodentiefe, Bodengründigkeit

- Nährstoffe in 0 bis $100 \mathrm{~cm}$ Bodentiefe: Ca, $\mathrm{Mg}, \mathrm{K}, \mathrm{C} / \mathrm{N}-$ Verhältnis (0 bis $10 \mathrm{~cm}$ Bodentiefe), Kationenaustauschkapazität

- Bodenversauerung bis $100 \mathrm{~cm}$ Bodentiefe: Aluminiumvorrat, Basensättigung, $\mathrm{pH}-$ Wert

- Luftverschmutzung: Stickoxid- und Ammoniumeintrag, S-Eintrag, AOT40-Werte.

Die statistischen Modelle wurden aus einer Kombination schrittweiser Methoden mit verschiedenen Informationskriterien ermittelt (Minimierung des Akaike-Informationskriteriums [AIC], des Bayes-Schwarz-Informationskriterium [BIC] und bestes Modell nach schrittweisen p-Wert-Schwellen). Es wurden nur Modelle gewählt, in denen die einzelnen ausgewählten Prädiktoren nicht stark miteinander korreliert waren (Korrelation <0.4) und eine p-Wert-Schwelle für die Selektion von $<0.1$ hatten.

\section{Resultate}

\section{Entwicklung der Kronenverlichtung}

In der Schweiz stieg der Anteil stark verlichteter Bäume bis Mitte der 1990er-Jahre stetig an (Abbildung 3). Danach können zwar grössere jährliche Schwankungen beobachtet werden, es zeigt sich jedoch kein langfristiger Trend. Die Entwicklung der Gesamtverlichtung und der Kronenverlichtung unbekannter Ursache verläuft insgesamt parallel. Demnach ist der Anteil der durch Schadursachen erklärbaren Verlichtung praktisch konstant geblieben. 


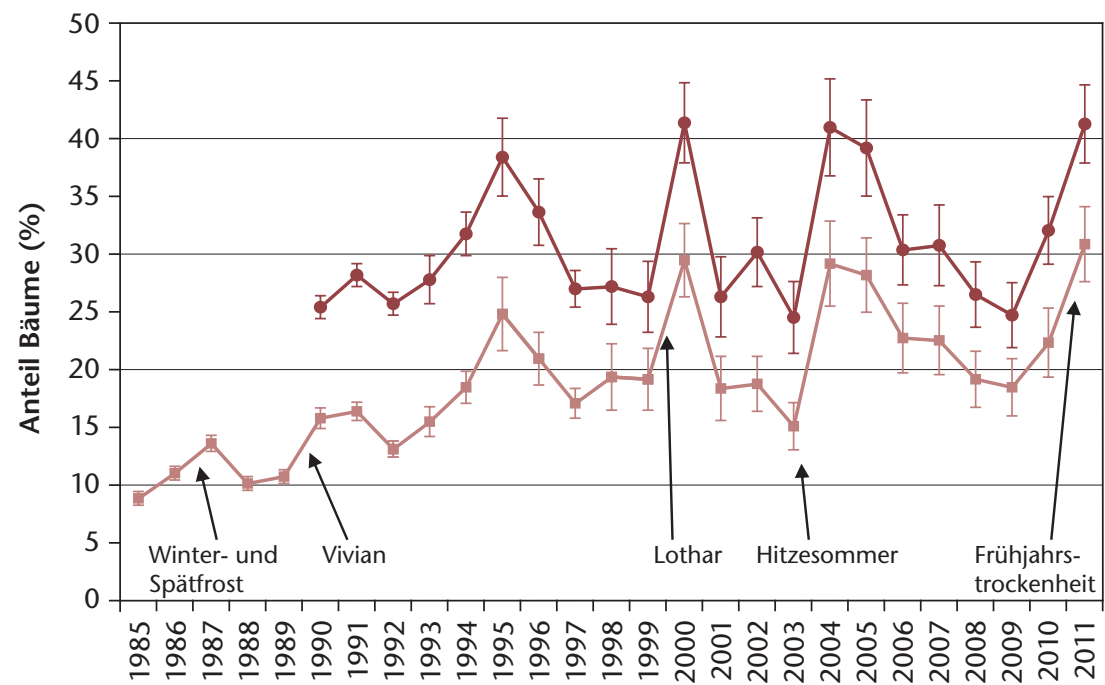

- Gesamtverlichtung $\quad-\quad-$ Kronenverlichtung unbekannter Ursache

Abb 3 Entwicklung des Anteils Bäume mit mehr als 25\% Gesamtverlichtung und Kronenverlichtung unbekannter Ursache seit 1985 (95\%-Vertrauensintervall). Wichtige klimatische Ereignisse mit nachfolgend hohem Anstieg der Verlichtung sind eingezeichnet.

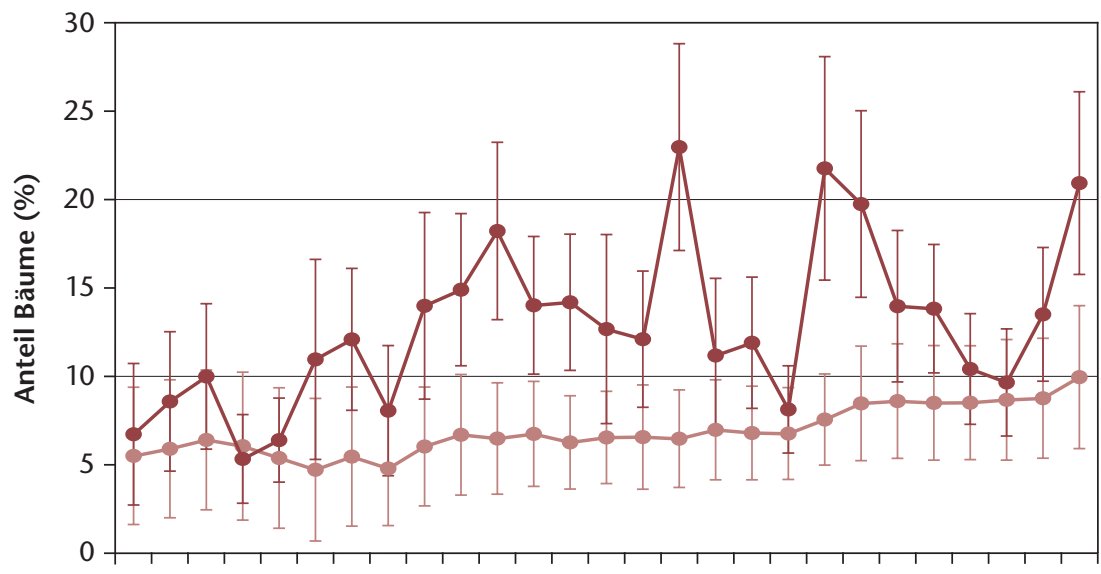

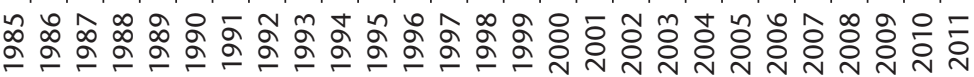

- Gesamtverlichtung $>25-95 \% \quad-$ - Stehend tot

Abb 4 Entwicklung des Anteils lebender Bäume mit >25-95\% Gesamtverlichtung und stehender toter Bäume seit 1985.

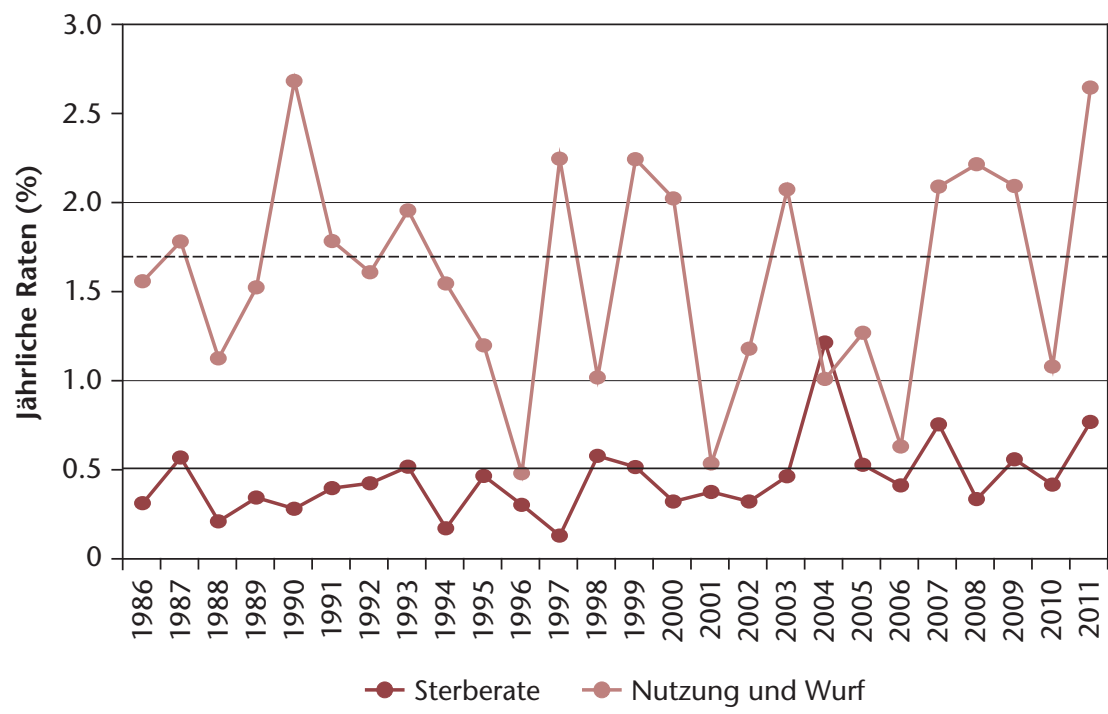

Abb 5 Entwicklung der Sterberaten und der Nutzungs- beziehungsweise Windwurfraten auf den Sanasilva-Flächen seit 1985 mit Angabe der langjährigen Mittelwerte. Statistische Fehler sind nicht dargestellt.
Einige auffällige Zunahmen der Kronenverlichtung von einem Jahr zum anderen fallen mit aussergewöhnlichen klimatischen Ereignissen zusammen. So zeigte sich jeweils ein Jahr nach dem Winter- und Spätfrost 1986/87, den Stürmen Vivian (Anfang 1990) und Lothar (Ende 1999), dem Hitzesommer 2003 und der extremen Frühjahrstrockenheit 2011 eine Zunahme der Kronenverlichtung (Abbildung 3).

In der Sanasilva-Inventur werden beim Anteil Bäume mit mehr als 25\% Kronenverlichtung alle stehenden Bäume berücksichtigt, also auch tote Bäume, die schon länger abgestorben sind. Dahinter stand zu Beginn der Erhebung die Überlegung, den möglichen Schädigungszustand der Wälder mit einer einzigen Aufnahme erfassen zu können. In Abbildung 4 ist der Anteil lebender Bäume mit >25-95\% Kronenverlichtung dem Anteil stehender toter Bäume gegenübergestellt. Seit 1992 hat sich der Anteil stehender toter Bäume von knapp 5\% auf fast 10\% im Jahr 2011 erhöht und trägt somit zu den höheren Kronenverlichtungswerten der letzten Jahre bei. Der Anteil lebender Bäume mit >25-95\% Gesamtverlichtung ist aber nur bis etwa 1995 angestiegen und schwankt seither zwischen 9 und 23\%.

Der Anstieg stehender toter Bäume im Schweizer Wald ist nicht auf einen Anstieg der jährlichen Sterberaten zurückzuführen (Abbildung 5). Die Sanasilva-Inventur zeigt seit 1985 weder eine steigende Mortalität noch eine steigende Nutzungsrate (inklusive Wind- und Schneewurf). Die jährlichen Sterberaten schwanken um $0.45 \%$ und die Nutzungsraten um 1.6\%. Ähnlich viele Bäume, wie durch Sterbeund Nutzungsrate wegfallen, wachsen jährlich in die Kluppschwelle ein. Durch die Reduktion des Netzes von $4 \times 4 \mathrm{~km}$ (knapp 700 Flächen) auf $16 \times 16 \mathrm{~km}$ (knapp 50 Flächen) erhöhte sich die statistische Unsicherheit stark. Seither schwanken die Mittelwerte von Jahr zu Jahr so stark, dass keine Unterschiede zwischen den einzelnen Jahren festgestellt werden können. Einzig nach dem Hitzesommer 2003 waren die Sterberaten höher als der langjährige Durchschnitt (über 1\%). Dieser Anstieg ist nicht nur auf den erhöhten Borkenkäferbefall der Fichte zurückzuführen, sondern betraf verschiedene Baumarten. Die Nutzungsraten im Rahmen der forstlichen Bewirtschaftung liegen in den meisten Jahren deutlich über den Absterberaten.

\section{Ursachen der Kronenverlichtung}

Tabelle 1 gibt eine Übersicht über die multivariate Auswertung der Sanasilva-Daten der Jahre 1993, 1994 und 1997. Dargestellt sind die besten Modelle pro abhängige Variable und Baumart. Für die Fichte erklären die Modelle zwischen 10 und 24\% der Streuung der Kronenverlichtung. Der wichtigste Einflussfaktor ist die Wasserverfügbarkeit im Boden. Sie ist in allen Modellen signifikant. Je kleiner die Wasserverfügbarkeit, desto deutlicher die Kronenverlich- 


\begin{tabular}{|c|c|c|c|c|c|c|c|c|c|c|c|c|c|c|c|c|c|c|c|c|}
\hline \multirow[t]{2}{*}{$\begin{array}{l}\text { Baum- } \\
\text { art }\end{array}$} & \multirow[t]{2}{*}{$\begin{array}{l}\text { Kronenverlichtung } \\
\text { und Verfärbung }\end{array}$} & \multirow[t]{2}{*}{$\mathbf{n}$} & \multirow[t]{2}{*}{$\begin{array}{l}\text { kor. } \\
r^{2}\end{array}$} & \multicolumn{2}{|c|}{ Klima } & \multicolumn{3}{|c|}{$\begin{array}{c}\text { Wasser- } \\
\text { verfügbarkeit }\end{array}$} & \multicolumn{5}{|c|}{ Nährstoffe } & \multicolumn{3}{|c|}{$\begin{array}{c}\text { Boden- } \\
\text { versauerung } \\
\end{array}$} & \multicolumn{4}{|c|}{ Luftschadstoffe } \\
\hline & & & & Nied & Temp & LAM & NWSK & Grue & $\mathrm{Ca}$ & Mg & K & $\mathrm{C} / \mathrm{N}$ & KAK & Al & BS & pH & NOx $_{x}$ & $\mathrm{NH}^{+}$ & $\mathrm{SO}_{2}$ & AOT40 \\
\hline \multirow[t]{4}{*}{ Fichte } & \multirow[t]{2}{*}{ Gesamtverlichtung } & 90 & 0.10 & & & & -- & & & & & & & & & & & & & \\
\hline & & 90 & 0.20 & ++ & & & -- & & & & & ++ & & & & & & & & \\
\hline & \multirow[t]{2}{*}{ Ursache unbekannt } & 90 & 0.16 & & -- & & -- & & & & & & & & & & & & & \\
\hline & & 90 & 0.24 & & -- & & -- & & & & & ++ & & & & ++ & ++ & & & \\
\hline \multirow[t]{5}{*}{ Buche } & \multirow[t]{3}{*}{ Gesamtverlichtung } & 54 & 0.18 & ++ & & & & & & & & & & & & & & & & \\
\hline & & 54 & 0.21 & ++ & & & & & & & - & & & & & & & & & \\
\hline & & 54 & 0.33 & & & ++ & & -- & & & -- & ++ & & -- & & ++ & + & & & \\
\hline & \multirow[t]{2}{*}{ Ursache unbekannt } & 54 & 0.15 & ++ & & & & & & & & & & & & & & & & \\
\hline & & 54 & 0.31 & & & ++ & & -- & & & -- & ++ & & -- & & & ++ & & & \\
\hline \multirow[t]{2}{*}{ Tanne } & Gesamtverlichtung & 35 & 0.21 & ++ & & & & & & -- & & & & & & & & & & \\
\hline & Ursache unbekannt & 35 & 0.21 & ++ & & & & & & & & & & & & -- & & & & \\
\hline \multirow{6}{*}{ Fichte } & \multirow[t]{2}{*}{ Farbton } & 89 & 0.10 & & -- & & & & & & & & & & & & & & & \\
\hline & & 89 & 0.13 & & -- & + & & & & & & & & & & & & & & \\
\hline & \multirow[t]{2}{*}{ Farbabweichung } & 89 & 0.06 & & -- & & & & & & & & & & & & & & & \\
\hline & & 89 & 0.09 & & -- & & & & & - & & & & & & & & & & \\
\hline & \multirow[t]{2}{*}{ Vergilbung } & 89 & 0.07 & & -- & & & & & & & & & & & & & & & \\
\hline & & 89 & 0.10 & & -- & & & & & - & & & & & & & & & & \\
\hline \multirow[t]{4}{*}{ Buche } & Farbton & 48 & 0.05 & & & & & & & & & + & & & & & & & & \\
\hline & Farbabweichung & 48 & 0.06 & + & & & & & & & & & & & & & & & & \\
\hline & \multirow[t]{2}{*}{ Vergilbung } & 48 & 0.24 & & & & -- & & & & -- & & & & & & & & + & - \\
\hline & & 48 & 0.23 & & & & -- & & & & -- & & & & & & + & & & -- \\
\hline \multirow[t]{4}{*}{ Tanne } & Farbton & 31 & - & & & & & & & & & & & & & & & & & \\
\hline & Farbabweichung & 31 & 0.13 & & & & & & & & -- & & & & & & & & & \\
\hline & \multirow[t]{2}{*}{ Vergilbung } & 31 & 0.18 & & & & & & & & -- & & & & & & & & & \\
\hline & & 31 & 0.21 & & & & & & & & -- & & & - & & & & & & \\
\hline
\end{tabular}

Tab 1 Signifikante Umweltfaktoren in den besten Modellen zur Erklärung der Gesamtverlichtung, der Kronenverlichtung unbekannter Ursache, der Belaubungsfarbe (Farbton), des Anteils Bäume mit Farbabweichung vom mittleren Farbton sowie der Vergilbung der Blätter oder Nadeln für Fichte, Buche und Tanne auf dem 8×8-km-Sanasilva-Netz (Daten der Jahre 1993, 1994 und 1997); positive Beziehung: $++(p-W e r t<0.05),+(p<0.1)$; negative Beziehung: $--(p<0.05),-(p<0.1) . n=$ Anzahl Flächen, kor. $r^{2}=$ nach Anzahl Parameter korrigierter Anteil der erklärten Streuung des Modells, Nied $=$ mittlerer Jahresniederschlag, Temp = Jahresmitteltemperatur, LAM = durchschnittlicher Trockenstress in den Sommermonaten, NWSK=nutzbare Wasserspeicherfähigkeit (bis $120 \mathrm{~cm}$ Bodentiefe), Grue =Bodengründigkeit, $\mathrm{Ca}, \mathrm{Mg}, \mathrm{K}=\mathrm{Ca}-\mathrm{-}, \mathrm{Mg}-\mathrm{-} \mathrm{K}$-Vorrat bis $1 \mathrm{~m}$ Bodentiefe, $\mathrm{C} / \mathrm{N}=\mathrm{C} / \mathrm{N}$-Verhältnis $(0-10 \mathrm{~cm}$ inklusive Humusauflage), $K A K=$ Kationenaustauschkapazität bis $1 \mathrm{~m}$ Bodentiefe, $A l=$ Aluminiumvorrat bis $1 \mathrm{~m}$ Bodentiefe, $B S=$ Basensättigung bis $1 \mathrm{~m}$ Bodentiefe, $p H=m i t t l e r e r$ Wert bis $1 \mathrm{~m}$ Bodentiefe, $\mathrm{NO}_{X}=$ berechnete Stickoxideinträge, $\mathrm{NH}^{+}=$berechnete Einträge von reduziertem Stickstoff, $\mathrm{SO}_{2}=\mathrm{Schwefeldioxideinträge,} A O T 40=\mathrm{Ozon}$ konzentration.

tung (Abbildung 6). Daneben scheinen die klimatischen Bedingungen einen wichtigen Einfluss zu haben. Je niedriger die Temperatur und je höher der Niederschlag, desto weniger waren die Fichten benadelt. Des Weiteren nimmt die Kronenverlichtung mit sinkendem $\mathrm{pH}$-Wert ab. In einem Modell korrelierten höhere N-Einträge mit der Kronenverlichtung: je höher das C/N-Verhältnis, desto stärker die Verlichtung, hingegen waren die Nährstoffvorräte im Boden in den Modellen nicht signifikant.

Für die Buche erklären die Modelle zwischen 15 und 33\% der Streuung der Kronenverlichtung. Wichtigste Variable ist der Niederschlag, wobei die Belaubung der Buche mit zunehmendem Niederschlag abnimmt. Dazu muss bemerkt werden, dass auf den Sanasilva-Flächen mit mindestens fünf lebenden Buchen der minimale jährlichen Niederschlag bei $980 \mathrm{~mm}$ lag. Das heisst, dass die Buche auf dem Sanasilva-Netz auf klimatisch trockenen
Standorten praktisch nicht vorkommt. Die höheren Kronenverlichtungen bei hohen $\mathrm{C} / \mathrm{N}$-Verhältnissen deuten auf eine höhere Kronenverlichtung bei schlechten Abbaubedingungen oder geringer N-Verfügbarkeit hin. Bei zwei Modellen sind die Kaliumversorgung und die Gründigkeit des Bodens negativ mit der Kronenverlichtung korreliert. Das heisst, es deutet sich ein positiver Effekt der Nährstoffverfügbarkeit an. Zwei Modelle zeigen, dass auf Standorten mit durchgehend ausreichender Wasserversorgung die Buche eher schlechter belaubt ist. Hier müsste näher untersucht werden, ob es sich bei Standorten mit schlecht belaubten Buchen um vernässte Standorte handelt. In zwei Modellen gibt es eine schwach signifikante positive Korrelation der N-Einträge mit der Kronenverlichtung.

Für die Tanne erklären die Modelle rund 20\% der Streuung der Kronenverlichtung. Auch hier sind Standorte mit hohen Niederschlägen eher nachtei- 


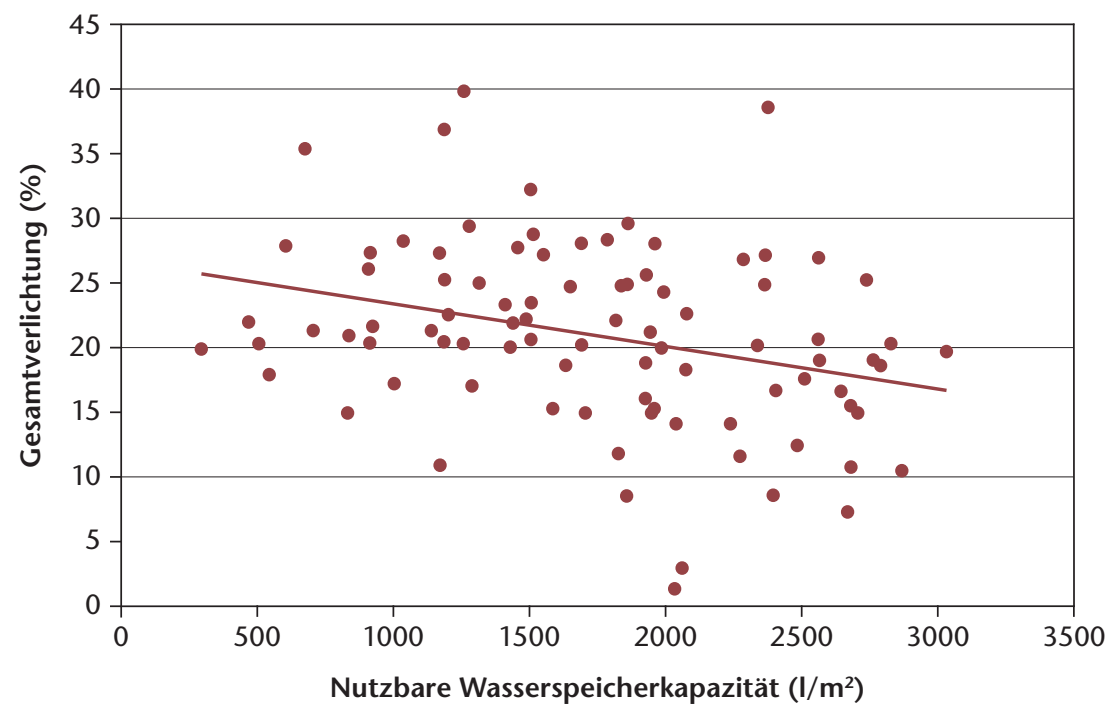

Abb 6 Beziehung zwischen der mittleren Gesamtverlichtung aller Fichten in den Jahren 1993 bis 1997 pro Probefläche und der nutzbaren Wasserspeicherfähigkeit.

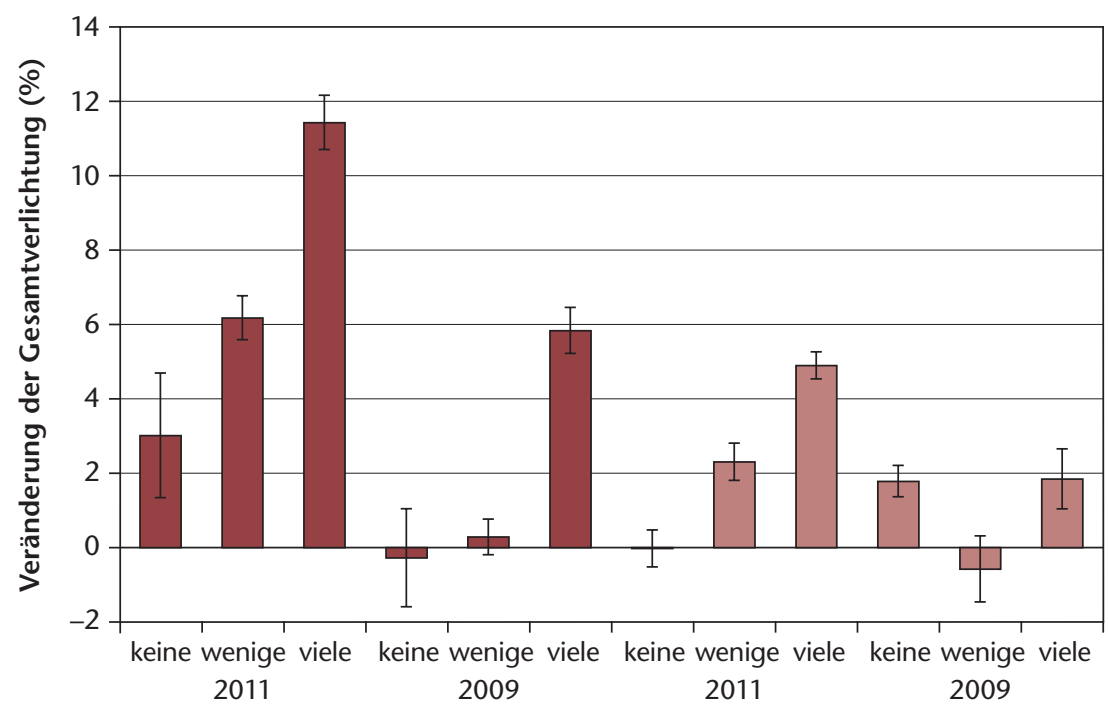

Abb 7 Änderung der Gesamtverlichtung in den Jahren 2011 und 2009 im Vergleich zum jeweiligen Vorjahr von Buchen (dunkle Säulen) und Fichten (helle Säulen) mit verschieden starkem Samenbehang (keine, wenige, viele) auf Sanasilva- und LWF-Flächen (95\%-Vertrauensintervall). in Abhängigkeit des Samenbehangs. 2011 war der Anstieg der Kronenverlichtung der Buche deutlich mit der Intensität der Samenproduktion gekoppelt. Bäume mit vielen Samen zeigten einen um 8\% höheren Anstieg, Bäume mit schwachem Samenbehang einen um 3\% höheren Anstieg als Bäume ohne Samen. Auch Fichten mit Samenbehang zeigten im Jahr 2011 einen signifikant stärkeren Anstieg der Kronenverlichtung (starker Samenbehang: +5\%, schwacher Samenbehang: +2\%). 2009 verzeichneten Buchen mit starkem Samenbehang einen Anstieg der Kronenverlichtung um knapp 6\%, jedoch zeigten Bäume mit schwachem Samenbehang keinen Anstieg der Kronenverlichtung. Bei der Fichte fand sich 2009 kein Zusammenhang zwischen Veränderung der Kronenverlichtung und Samenbehang.

\section{Ursachen der Farbe der Belaubung}

Für die Fichte können die Modelle lediglich zwischen 6 und 13\% der Streuung der Nadelfarbe erklären. In allen Modellen hat die mittlere Jahrestemperatur einen signifikanten Einfluss. Bei niedrigen Temperaturen sind die Fichten weniger dunkelgrün und mehr Fichten weisen vergilbte Nadeln auf. Zwei Modelle zeigen: Je besser die Nährstoffversorgung mit Magnesium, umso seltener treten vergilbte Bäume auf.

Bei der Buche erklären die Modelle zwar nur zwischen 4 und 6\% der Streuung des Farbtons, aber 20 bis 24\% des Anteils der Bäume mit Vergilbung. Die Buchenblätter scheinen bei schlechten klimatischen Bedingungen (hoher Niederschlag oder hohes C/N-Verhältnis) ins Gelbgrüne zu tendieren. Der Anteil der Bäume mit Vergilbungen scheint vor allem auf Standorten mit niedrigerer Wasserspeicherfähigkeit und schlechterer Kaliumversorgung anzusteigen. Zwar zeigen einige Modellen auch leicht signifikante Einflüsse von Lufteinträgen. Es gibt etwas mehr gelbe Blätter bei erhöhtem N- und S-Eintrag, aber weniger bei erhöhten Ozonkonzentrationen. Die Beziehungen sind aber wenig eindeutig.

Kein Modell kann die generelle Kronenfarbe der Tannen erklären. Hingegen nimmt der Anteil Tannen mit Grünabweichungen oder Vergilbungen mit der Kaliumverfügbarkeit im Boden ab, wobei zwischen 13 und 21\% der Abweichungen erklärt werden können.

\section{Diskussion}

\section{Kronenverlichtung und Sterberate}

Eine Veränderung in der Methodik, welche den bis 1995 beobachteten Anstieg der Kronenverlichtung erklären würde, kann ausgeschlossen werden. Vergleiche von zwischen 1986 und 1997 gemachten Dias (auf sogenannten Fotoparcours) mit im Feld beurteilten Bäumen gaben keine Hinweise 
auf eine veränderte Beurteilung der Kronenverlichtung (Dobbertin et al 2004).

Ein Vergleich mit anderen Ländern Europas zeigt, dass in Teilen Westeuropas die Kronenverlichtung bis Ende der 1990er-Jahre anstieg und seitdem auf einem ähnlichen Niveau verharrt (Goudet 2011; Waldzustandsberichte $2011^{2}$ für Baden-Württemberg, Niedersachsen, Nordrhein-Westfalen, Rheinland-Pfalz). Im Gegensatz dazu fand in den östlichen Bundesländern Deutschlands seit Beginn der Erhebungen im Jahr 1991 ein Rückgang der Kronenverlichtung bis etwa 2000 statt (Waldzustandsberichte 2011 für Thüringen ${ }^{2}$ und Sachsen ${ }^{3}$ ). Dies deutet auf einen Einfluss der Verminderung der Luftschadstoffe nach der politischen Wende hin. Ein stärkerer Anstieg der Kronenverlichtung wurde in den Jahren 1990, 2004, 2011 und in geringerem Mass im Jahr 2000 verzeichnet. Die Buche zeigte 2011 in allen Bundesländern und 2004 in den meisten Regionen einen deutlichen Anstieg der Kronenverlichtung, während bei den anderen Baumarten das Bild nicht so einheitlich ist. Als Erklärung liegt auf den ersten Blick der Einfluss von Witterungsextremen wie der Stürme Vivian und Wiebke im Februar 1990, Lothar und Martin im Dezember 1999, des extrem trockenen Sommers 2003 und der Frühjahrstrockenheit 2011 nahe. Wenn jedoch durch die Stürme beispielsweise Baumkronen beeinträchtigt wurden, sind die Resultate der verschiedenen Erhebungen sehr uneinheitlich.

Die jährlichen Mortalitätsraten liegen in der Schweiz etwas über denjenigen in den Nachbarländern (Neumann \& Stemberger 1990, Renaud \& Nageleisen 2005, Eichhorn et al 2008). Dies liegt aber zum Teil daran, dass in der Schweiz auch unterständige Bäume erhoben werden, die aufgrund der erhöhten Konkurrenz durch Nachbarbäume häufiger absterben. Bäume höherer sozialer Stellung sterben auch im Schweizer Wald seltener ab. Der Anstieg der Sterberate nach dem Hitzesommer 2003 wurde auch in Deutschland und in Frankreich beobachtet (Eichhorn et al 2008, Renaud \& Nageleisen 2005) und legt eine Schwächung der Bäume durch die Trockenheit und Hitze nahe. Auf der LWF-Fläche in Visp (Wallis) konnte ein eindeutiger Zusammenhang zwischen Sommertrockenheit und Absterben der Föhren im Folgejahr belegt werden (Dobbertin \& Rigling 2006).

Aus der Beobachtung, dass zwar die Kronenverlichtung zwischen 1985 und 1995 angestiegen ist und seitdem auf einem höheren Niveau verharrt, die jährlichen Sterbe- und Nutzungsraten seit 1985 jedoch keinen Anstieg verzeichnen, darf nicht geschlossen werden, dass es keinen Zusammenhang zwischen der Kronenverlichtung und dem Absterberisiko eines Baumes gibt. Untersuchungen auf den Sanasilva- und LWF-Flächen belegen eine klare Beziehung zwischen der Kronenverlichtung eines Baumes und seiner Überlebenswahrscheinlichkeit
(Dobbertin \& Brang 2001). Ob ein Baum letztendlich aber abstirbt, hängt von einer Vielzahl weiterer Faktoren ab.

\section{Ursachen des Kronenzustands}

Bei der Diskussion der Ergebnisse der Modellauswertung muss berücksichtigt werden, dass das Baumalter nicht einbezogen werden konnte, da es aufgrund der meist ungleichaltrigen Bestände im Schweizer Wald nicht genau bestimmt werden kann. Es ist jedoch bekannt, dass das Baumalter besonders bei den Nadelbäumen ein sehr wichtiger Faktor des Kronenzustandes ist (Neumann 1989b, Klap et al 2000). Mit zunehmendem Baumalter nimmt in der Regel die Kronenverlichtung zu (Klap et al 2000, Ewald 2005, Musio et al 2007, Seidling 2007). Trotz der fehlenden Informationen zu Baumalter in den Modellen können zwischen 10 und 30\% der Kronenverlichtung und zwischen 6 und 24\% der Streuung der Farbabweichungen der Nadeln und Blätter erklärt werden. Klap et al (2000) haben in einer europaweiten Auswertung der Level-I-Flächen einen etwas grösseren Anteil der Kronenverlichtung erklären können, als es in unserer Studie gelang. Die wichtigsten erklärenden Variablen sind der Unterschied zwischen den Ländern und das Baumalter.

\section{Klimatische Einflüsse}

Generell kann festgehalten werden, dass in unserer Studie ungünstige klimatische Bedingungen (niedrige Temperaturen und hohe Niederschläge) mit einem schlechteren Kronenzustand korrelieren. Dies ist auf den ersten Blick überraschend, da erwartet wird, dass der Kronenzustand eher auf Standorten mit geringeren Niederschlägen schlechter sein sollte. Es muss aber festgehalten werden, dass bei den Baumarten Buche und Tanne Flächen mit geringen Niederschlägen fehlten. Seidling (2007) zeigte zwar für Deutschland, dass die Kronenverlichtung der meisten Baumarten nach Trockenheit und Sommerhitze (besonders nach dem Hitzesommer 2003) anstieg. Für einzelne Baumarten (Eiche und Buche) führten aber auch erhöhte Niederschläge zu einem Anstieg der Kronenverlichtung. Der für die Buche und Tanne gefundene Einfluss des Niederschlags ist deshalb kein Widerspruch zur Trockenheitshypothese.

Dass die Kronenverlichtung mit der Höhe über Meer ansteigt, ist schon früher beobachtet worden (Abbildung 8; Brang 1998). Die Referenzfotos berücksichtigen die spezielle Verzweigungsstruktur der Bäume in Hochlagen nur ungenügend. Für die Baumarten Fichte und Tanne wurde dies mithilfe von seehöhenspezifischen Referenzfotos zu kompensieren versucht (Fichte: Unterscheidung in Kamm-, Bürsten- und Plattenfichte, Tanne: Unterscheidung

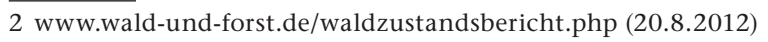




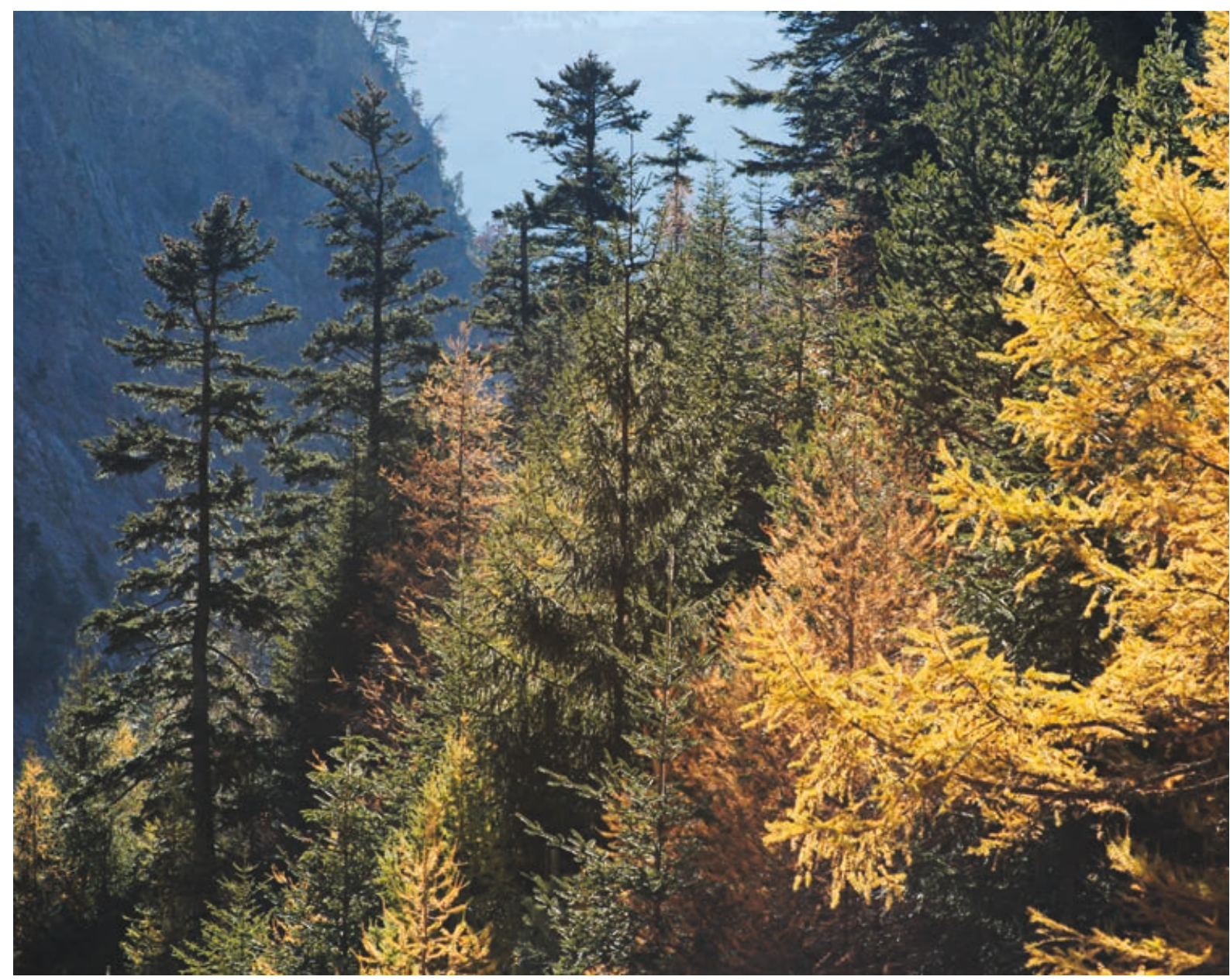

Abb 8 Weisstannen (links im Bild) im Bietschtal, Wallis, auf rund 1200 m ü. M. Mit der Meereshöhe nimmt die Kronenverlichtung der Bäume in der Regel zu. Foto: Brigitte Wolf

in Tieflagen- und Gebirgstyp; Müller \& Stierlin 1990), was aber offensichtlich nicht ausreicht. Allerdings steigt das durchschnittliche Baumalter mit der Meereshöhe ebenfalls an, was den Anstieg der Kronenverlichtung teilweise erklären könnte.

Schlechtere klimatische Bedingungen bedeuten aber auch geringere Abbauraten der Streu und damit höhere C/N-Verhältnisse in Streu und Oberboden. In unserer Studie ist die Kronenverlichtung von Fichten und Buchen auf Standorten mit hohem C/N-Verhältnis ausgeprägter. Die verstärkte Kronenverlichtung dieser Baumarten könnte also auch durch eine schlechte N-Versorgung bedingt sein.

\section{Wasserverfügbarkeit}

Die Kronenverlichtung der Fichte und der Anteil Buchen mit Abweichung vom Grünfarbton sind auf Standorten mit geringer Wasserspeicherfähigkeit grösser, während die Buchen auch auf sehr feuchten Standorten mit stärkerer Kronenverlichtung reagieren. Zierl (2004) hatte mithilfe eines ökophysiologischen Wasserhaushaltsmodells für die Sanasilva-Flächen keine räumliche Beziehung zwischen Kronenverlichtung und Wasserhaushalt gefunden, wohl aber eine Beziehung zwischen modelliertem Wasserhaushalt und dem Anstieg der Kronenverlichtung im Folgejahr. Diese Beziehung war im Gegen- satz zu unserer Studie für die Laubbäume stärker ausgeprägt als für die Nadelbäume. Webster et al (1996) fanden für die Sanasilva-Flächen mit anderen Auswertungsmethoden und Daten für die Fichte und in geringem Masse für die Tanne eine erhöhte Kronenverlichtung mit Abnahme der Wasserspeicherfähigkeit des Bodens. Für die Buche gab es keine solche Beziehung. Auch Ewald (2005) fand für die Fichte auf Standorten der Kalkalpen eine Korrelation zwischen Kronenverlichtung und Wasserverfügbarkeit im Boden.

\section{Nährstoffe}

Bei der Buche und der Tanne gibt es eine Tendenz dazu, dass die Kronenverlichtung und der Anteil Bäume mit vergilbten Nadeln oder Blätter mit abnehmender Magnesium- und Kaliumversorgung abnimmt. Bei Fichten fanden wir nur für die Magnesiumversorgung und den Anteil vergilbter Bäume einen schwachen Zusammenhang. Verschiedene Studien haben gezeigt, dass die Nährstoffversorgung der Bäume mit der Kronenverlichtung (Flückiger \& Braun 1995, Augustin et al 2005, Musio et al 2007) oder der Kronenverfärbung (Musio et al 2004) korreliert ist. Je besser die Versorgung mit Nährstoffen (vor allem mit $\mathrm{Mg}, \mathrm{K}$ und $\mathrm{P}$ ), desto geringer ist die Kronenverlichtung und desto grüner sind die Nadel- 
oder Blattfarben. Ewald (2005) konnte zeigen, dass der Kronenzustand der Fichte in den bayerischen Kalkalpen vor allem von der Nährstoffversorgung im Boden abhing (neben der Wasserverfügbarkeit). In einem Experiment auf einem schlecht mit Nährstoffen versorgten Kalkboden bei Alvaneu (Graubünden) konnte durch Mineraldüngerzugabe die Kronenverlichtung von Fichten deutlich reduziert werden (Flückiger \& Braun 1995, Joos 1997).

\section{Luftschadstoffe}

Die Variablen der Bodenversauerung können in unserer Studie in keinen eindeutigen Zusammenhang mit dem Kronenzustand gebracht werden. Bei den Luftschadstoffen deutet sich allenfalls bei der Fichte und der Buche ein negativer Zusammenhang zwischen Kronenverlichtungen und oxidierten NEinträgen an. Klap et al (2000) hatten in ihrer europaweiten Studie zum Teil negative Korrelationen zwischen $\mathrm{SO}_{2}$ - und $\mathrm{NO}_{\mathrm{X}}$-Konzentrationen und der Kronenverlichtung der Buche und Eiche gefunden. Allerdings erklärten diese nur einen kleinen Teil der gesamten Streuung der Kronenverlichtung. In einer Studie zur Veränderung der Kronenverlichtung auf den Sanasilva-Flächen hatten Innes et al (1997) einen negativen Effekt der $\mathrm{SO}_{2}$-Konzentration auf die Entwicklung der Kronenverlichtung der Waldbäume bis 1996 gefunden, nicht aber für die $\mathrm{NO}_{\mathrm{X}}$-Belastung. Auf den LWF-Flächen fanden Thimonier et al (2010) dagegen, dass Nadelbäume mit höherem $\mathrm{N}$-Anteil in den Nadeln eine geringere Kronenverlichtung aufwiesen. Da gleichzeitig eine Beziehung zwischen N-Eintrag und N-Gehalt in den Nadeln gefunden wurde, konnte in der Studie kein negativer Effekt der N-Belastung auf den Kronenzustand belegt werden.

Zwischen Ozonkonzentration und Kronenzustand können in unserer Studie keine signifikant negativen Korrelationen gefunden werden. Ein Grund dafür ist sicherlich, dass die Ozonkonzentrationen von Jahr zu Jahr variieren und deshalb die Betrachtung einzelner Jahre nicht ausreicht, um eine solche Beziehung nachweisen zu können. Innes et al (1997) und Zierl (2002) fanden, dass auf den Sanasilva-Flächen erhöhte Ozonkonzentrationen mit ansteigenden Kronenverlichtungen korrelierten. Ähnliches für Buchen fanden auch Bussotti et al (2009) und Ferretti et al (1998, 2007). In allen Studien konnte die Ozonbelastung jedoch nur einen sehr geringen Teil der Veränderung der Kronenverlichtung erklären.

\section{Samenbildung}

In der Sanasilva-Inventur wurde 2011 bei den Baumarten Buche, Fichte, Tanne und Ahorn der höchste bisher registrierte Anteil fruktifizierender Bäume beobachtet. Dasselbe Phänomen wurde bei der Buche und Fichte auch in Frankreich und in deutschen Bundesländern beobachtet (Goudet 2011;
Waldzustandsberichte $2011^{2}$ z.B. für Baden-Württemberg, Niedersachsen, Nordrhein-Westfalen, Rheinland-Pfalz oder Sachsen ${ }^{3}$ ). Zumindest für die Buche ist bekannt, dass die Kronenverlichtung mit starkem Samenbehang ansteigt (Schmitt 1991). Auch Seidling (2007) fand in seiner Studie, dass der Anstieg der Kronenverlichtung bei der Buche in Deutschland im Jahr 2004 auch auf das Mastjahr 2004 zurückzuführen war. Praktisch identische Ergebnisse wurden in Rheinland-Pfalz und Baden-Württemberg gefunden. Bei der Buche erklärt sich die erhöhte Kronenverlichtung zum einem durch die aufgrund der Blütenbildung geringere Anzahl Blätter und zum anderen durch die im Mastjahr reduzierte Blattgrösse. Bei der Fichte hingegen sind die Nadellängen im Mastjahr nicht offensichtlich reduziert, und die relativ geringe Anzahl Zapfen kann nur einen gewissen Teil der Neubildung der Triebe verhindern. Beprobungen von Zweigen im Jahr 2011 auf LWF-Flächen zeigten jedoch, dass an einzelnen Zweigen männliche Blüten in ganz erheblichem Ausmass die zweigbildenden Knospen ersetzten. Dadurch gab es 2011 kaum neue Nadeln an diesen Zweigen. Dieses Phänomen muss aber noch genauer untersucht werden.

Im Jahr 2009 - ebefalls ein gutes Samenjahr war der Effekt für die Buche weniger stark und für die Fichte nicht vorhanden. Die Ergebnisse verdeutlichen, dass der Einfluss des Samenbehangs nicht in jedem Mastjahr gleich gross ist. Das Beispiel des Einflusses der Samenbildung auf die Belaubung der Waldbäume macht aber klar, dass eine erhöhte Kronenverlichtung keinesfalls direkt mit einer Schädigung des Baumes gleichgesetzt werden darf. Die in den letzten Jahren beobachtete Zunahme der Mastjahre, vor allem bei der Buche, und ihr Einfluss auf die Belaubung bedürfen deshalb einer erhöhten Aufmerksamkeit.

\section{Bewertung und Ausblick}

Die Fortführung der Waldzustandsinventuren mittels Kronenansprachen ist heute umstritten. Das liegt zum einen daran, dass die Belaubung des Einzelbaums trotz des Versuchs, Computerprogramme einzusetzen (Mizoue \& Dobbertin 2003), nicht wirklich messbar ist und deshalb der Subjektivität des Beobachters unterliegt. Zum anderen ist die Kronenverlichtung (aber auch die Farbe der Belaubung) als Indikator der Baumvitalität oder gar der Waldgesundheit umstritten. Schliesslich stellt sich auch die Frage, ob die Kronenverlichtung als Indikator der negativen Auswirkungen von Luftschadstoffen auf den Wald geeignet ist.

Wie in der vorliegenden Studie zur SanasilvaInventur gezeigt wurde, können Trends und jährli-

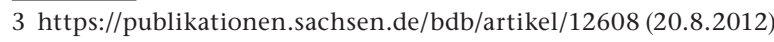




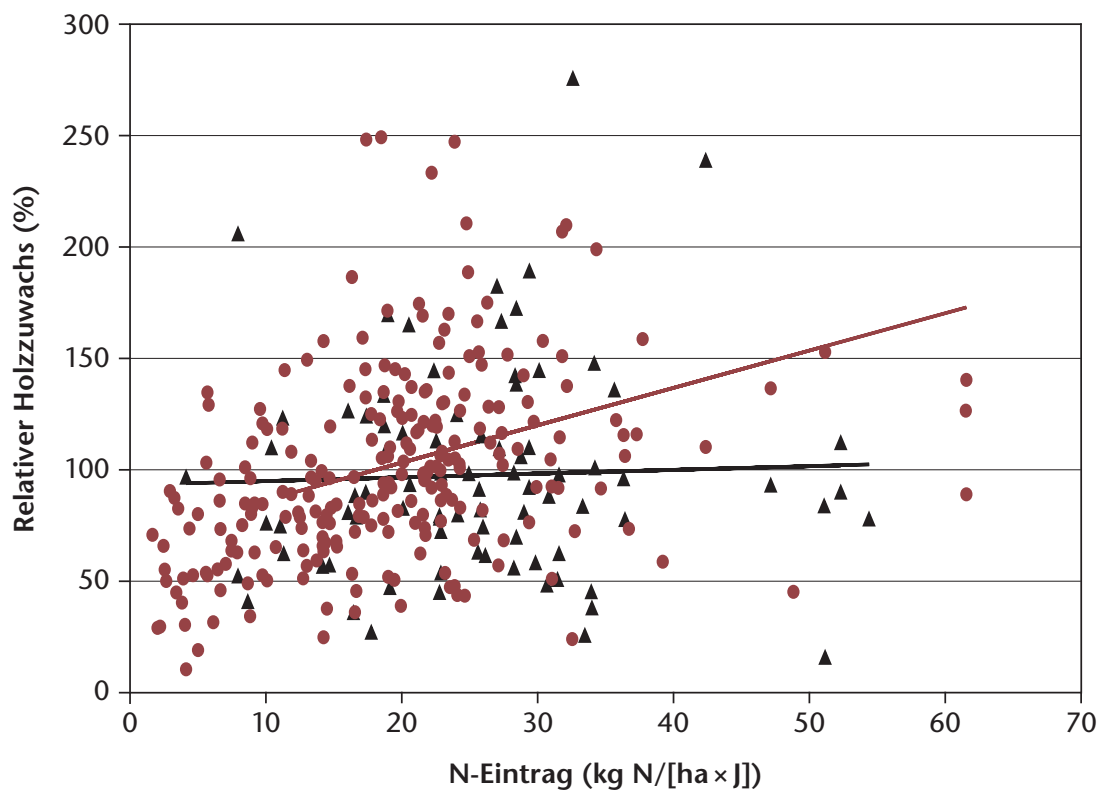

$\Delta$ Viel Bodenstickstoff, gemessener Zuwachs _ - Viel Bodenstickstoff, modelliert - Wenig Bodenstickstoff, gemessener Zuwachs — Wenig Bodenstickstoff, modelliert

Abb 8 Relativer Holzzuwachs auf langfristigen Untersuchungsflächen in den Jahren 1994 bis 1999 (berechnet nach einem Modell für Bestandesalter, Bestandesdichte und Ertragsklasse) in Abhängigkeit vom modellierten jährlichen N-Eintrag und vom C/N-Verhältnis im Oberboden (Solberg et al 2009).

che Veränderungen in der Kronenverlichtung trotz Ungenauigkeiten bei der Ansprache durchaus statistisch ausgewertet und interpretiert werden. Voraussetzung dafür ist allerdings eine strenge Qualitätssicherung, um systematische Abweichungen oder Veränderungen in der Erhebungsmethodik zu verhindern. Bei der Interpretation von Veränderungen ist bei einer geringen Stichprobenzahl zudem Vorsicht geboten.

Wie viele Studien gezeigt haben, gibt es $\mathrm{Zu}$ sammenhänge zwischen Standortvariablen, Klima und Kronenzustand der Bäume. Die Frage, ob die visuellen Kronenansprachen (Kronenverlichtung und Nadel-/Blattverfärbung) als Indikator der Baumvitalität geeignet sind, ist deshalb weniger eine Frage der Erhebungen als vielmehr der Begriffe geschädigt, gesund oder vital, welche immer eine Interpretation aus menschlicher Sicht darstellen. So sind zum Beispiel Bäume auf schlecht mit Nährstoffen versorgten Hochlagenstandorten oder auf Standorten mit geringer Wasserversorgung (zum Beispiel im Wallis) nicht stärker geschädigt oder geschwächt als Bäume auf anderen Standorten. Der Kronenzustand beschreibt den typischen Zustand der Bäume an diesen Standorten. Durch Zugabe von Dünger (Joos 1997) oder Wasser (Dobbertin et al 2010) kann zwar die Kronenverlichtung reduziert werden, aber es stellt sich die Frage, ob die Bäume deshalb gesünder sind. Desgleichen sind auch Bäume mit hohem Samenbehang nicht geschädigt oder weniger vital.

Die Verwendung nur eines Indikators, um den Zustand und die Entwicklung des Waldes zu be- schreiben, ist ebenfalls heikel. Das zeigen die Auswertungen der Sanasilva-Daten. Obwohl beispielsweise ein Zusammenhang zwischen der Kronenverlichtung und der Absterbewahrscheinlichkeit (Neumann \& Stemberger 1990, Dobbertin \& Brang 2001) beziehungsweise dem Zuwachs des Einzelbaums (Solberg \& Tveite 2000, Dobbertin 2005) besteht, bedeutet ein regionaler oder nationaler Anstieg der Kronenverlichtung nicht automatisch einen Anstieg der Mortalität oder eine Reduktion des Zuwachses. Zum einen ist in der Sanasilva-Inventur die Mortalität nicht gleichzeitig mit der Kronenverlichtung angestiegen, zum anderen hat das Wachstum der Waldbäume in der Schweiz im gleichen Zeitraum sogar zugenommen (Zingg 1996, Köhl 1996, Bräker 1996). Verschiedene Studien haben gezeigt, dass erhöhte Stickstoffeinträge auf mässig stickstoffversorgten Böden das Baumwachstum erhöht haben, während es auf besser versorgten Böden unverändert blieb (Kahle et al 2008, Solberg et al 2009, Laubhann et al 2009). Abbildung 8 verdeutlicht diesen Zusammenhang zwischen Baumwachstum und N-Eintrag für die Jahre 1994 bis 1999 auf den Level-II-Flächen von ICP Forests. Deshalb sind die Bäume mit erhöhtem Wachstum aber nicht automatisch als gesünder oder vitaler anzusehen.

Es stellt sich die Frage, inwieweit der Kronenzustand als Indikator für die Auswirkungen der Luftschadstoffe geeignet ist. Zwar zeigen einige Studien signifikante Beziehungen zwischen Kronenzustand und Schadstoffkonzentrationen in der Luft, doch erklären Letztere den Kronenzustand meist viel weniger gut als klimatische, standörtliche oder biologische Faktoren. Zu Zeiten der akuten Rauchgasschäden war dies sicherlich anders. Die Beziehung zwischen Nährstoffversorgung und Kronenzustand legt nahe, dass langfristige, durch Einträge aus der Luft verursachte Veränderungen der Nährstoffversorgung sich auch langfristig auf den Kronenzustand auswirken. Doch kann ein solcher Einfluss besser über die unmittelbare Messung der Einträge und der Nährstoffversorgung in Boden, Nadeln und Blättern gemessen werden (Thimonier et al 2012, Braun et al 2012, Graf Pannatier et al 2012, alle dieses Heft). Schleichende langfristige Veränderungen können nur schlecht über die Erhebung der Kronenverlichtung erfasst werden.

Abschliessend kann aber gesagt werden, dass der Kronenzustand auch heute noch ein brauchbarer Indikator des Waldzustands ist. Er sollte aber auf jeden Fall nur im Zusammenhang mit anderen Indikatoren wie Sterberaten, Wachstum oder Nährstoffversorgung interpretiert werden. Gerade in Zeiten der globalen Veränderung (Nutzung, Klima und Zusammensetzung der Atmosphäre) braucht es langfristig erhobene und vergleichbare Indikatoren.

Eingereicht: 23. Juli 2012, akzeptiert (mit Review): 1. August 2012 


\section{Literatur}

AUGUSTIN S, ACHERMANN B (2012) Deposition von Luftschadstoffen in der Schweiz: Entwicklung, aktueller Stand und Bewertung. Schweiz Z Forstwes 163: 323-330. doi: 10.3188/ szf.2012.0323

AUGUSTIN S, BOLTE A, HOLZHAUSEN M, WOLFF B (2005) Exceedance of critical loads of nitrogen and sulphur and its relation to forest conditions. Eur J Forest Res 124: 289-300.

BRANG P, EDITOR (1998) Sanasilva-Bericht 1997. Zustand und Gefährdung des Schweizer Waldes - eine Zwischenbilanz nach 15 Jahren Waldschadenforschung. Birmensdorf: Eidgenöss Forsch.anstalt Wald Schnee Landschaft, Bericht 345. 102 p.

BRÄKER OU (1996) Growth trends of Swiss forests: tree-ring data. Case study Toppwald. In: Spiecker H, Mielikäinen K, Köhl M, Skovsgaard JP, editors. Growth trends in European forests. Berlin: Springer. pp. 199-217.

BRAUN S, RIHM B (2012) Ozonbelastung von Waldbäumen in der Schweiz und damit verbundene Wachstumseinbussen. Schweiz Z Forstwes 163: 383-388. doi: 10.3188/szf.2012.0383

BRAUN S, RIHM B, FLÜCKIGER W (2012) Stickstoffeinträge in den Schweizer Wald: Ausmass und Auswirkungen. Schweiz Z Forstwes 163: 355-362. doi: 10.3188/szf.2012.0355

BUSSOTTI F ET AL (2009) Quality assurance and measurement errors in monitoring tree crown conditions in Italy. J Environ Monitor 11: 769-773.

DOBBERTIN M (2005) Tree growth as indicator of tree vitality and of tree reaction to environmental stress: a review. Eur J Forest Res 124: 319-333.

DOBBERTIN M, BRANG P (2001) Crown defoliation improves tree mortality models. Forest Ecol Manag 141: 271-284.

DOBBERTIN M ET AL (2010) Effect of irrigation on needle morphology, shoot and stem growth in a drought-exposed Pinus sylvestris forest. Tree Physiol 30: 346-360.

DOBBERTIN M, HUG C, WALDNER P (2009) Kronenverlichtung, Sterberaten und Wachstum in Langzeitstudien - welche Indikatoren beschreiben den Waldzustand am besten? In: WSL. Langzeitforschung für eine nachhaltige Waldnutzung. Birmensdorf: Eidgenöss Forsch.anstalt Wald Schnee Landschaft, Forum für Wissen. pp. 7-20.

DOBBERTIN M, RIGLING A (2006) Pine mistletoe (Viscum album ssp austriacum) contributes to Scots pine (Pinus sylvestris) mortality in the Rhone valley of Switzerland. For Pathol 36: 309-322.

EICHHORN J ET AL (2008) Untersuchungen zur Trockenheitstoleranz der Buche am Beispiel des witterungsextremen Jahres 2003. In: NW-FVA. Ergebnisse angewandter Forschung zur Buche. Nordwestdeutschen Forstlichen Versuchsanstalt Göttingen, Beiträge 3: 109-134.

EWALD J (2005) Ecological background of crown condition, growth and nutritional status of Picea abies (L.) Karst. in the Bavarian Alps. Eur J Forest Res 124: 9-18.

FERRETTI M, BARATOZZI L, CENNI E, COZZI A, SAVINI P (1998) Crown transparency of beech (Fagus sylvatica $\mathrm{L}$.) in the northern Apennines (Italy). Status, changes and relationships with site characteristics and other indices of tree condition. Chemosphere 36: 1037-1042.

FERRETTI M, CALDERISI M, BUSSOTTI F (2007) Ozone exposure, defoliation of beech (Fagus sylvatica L.) and visible foliar symptoms on native plants in selected plots of South-Western Europe. Environ Pollut 145: 644-651.

FLÜCKIGER W, BRAUN S (1995) Revitalization of an alpine protective forest by fertilization. Plant Soil 168: 481-488.

GOUDET M (2011) Bilan de la santé des forêts 2011. Le réseau systématique de suivi des dommages forestiers en 2011. Paris: Département de la santé des forêts.
GRAF PANNATIER E, THIMONIER A, SCHMITT M, WALDNER P, WALTHERT L (2012) Impacts des dépôts atmosphériques acides sur l'eau des sols forestiers. Schweiz Z Forstwes 163: 363-373. doi: 10.3188/szf.2012.0363

INNES JL, GHOSH S, SCHWYZER A (1996) A method for the identification of trees with unusually colored foliage. Can J For Res 26: 1548-1555.

INNES JL, GHOSH S, DOBBERTIN M, REBETEZ M, ZIMMERMANN $S$ (1997) Kritische Belastung und die Sanasilva-Inventur. In: WSL. Säure- und Stickstoffbelastungen - ein Risiko für den Schweizer Wald? Birmensdorf: Eidgenöss Forsch.anstalt Wald Schnee Landschaft, Forum für Wissen. pp. 73-83.

JOOS K (1997) lonengehalt und elektrische Leitfähigkeit im Splintholz, Zuwachsleistung und Nadelverlust bei Fichte unter Berücksichtigung ihrer Nährstoffversorgung. Zürich: Eidgenöss Techn Hochschule, Professur Holzwissenschaften, wissenschaftl Ber 1. 217 p.

KAHLE ET AL (2008) Causes and consequences of forest growth trends in Europe. Results of the recognition project. Brill: European Forest Institute, Research Project 21. $261 \mathrm{p}$.

KLAP JM, VOSHAAR JHO, DE VRIES W, ERISMAN JW (2000) Effects of environmental stress on forest crown condition in Europe. Part IV: Statistical analysis of relationships. Water Air Soil Poll 119: 387-420.

KÖHL M (1996) Growth patterns in forests of the canton of Berne, Switzerland, based on inventory data. In: Spiecker $\mathrm{H}$, Mielikäinen K, Köhl M, Skovsgaard JP, editors. Growth trends in European forests. Berlin: Springer. pp. 219-237.

LAUBHANN D, STERBA H, REINDS GJ, DE VRIES W (2009) The impact of atmospheric deposition and climate on forest growth in European monitoring plots: An individual tree growth model. Forest Ecol Manage 258: 1751-1761.

MIZOUE N, DOBBERTIN M (2003) Detecting differences in crown transparency assessments between countries using the image analysis system CROCO. Environ Monit Assess 89: 179-195.

MÜLLER E, STIERLIN HR (1990) Sanasilva-Kronenbilder mit Nadelund Blattverlustprozenten. Birmensdorf: Eidgenöss Forsch.anstalt Wald Schnee Landschaft, 2. ed. $129 \mathrm{p}$.

MUSIO M ET AL (2004) Predicting magnesium concentration in needles of Silver fir and Norway spruce - a case study. Ecol Model 179: 307-316.

MUSIO M, VON WILPERT K, AUGUSTIN NH (2007) Crown condition as a function of soil, site and tree characteristics. Eur J Forest Res 126: 91-100.

NEUMANN M (1989A) Zu Fragen der Waldzustandserfassung durch grossräumige Inventuren. Cent.bl gesamte Forstwes 106: $161-178$.

NEUMANN M (1989B) Einfluss von Standortsfaktoren auf den Kronenzustand. In: Bucher JB, Bucher-Wallin I, editors. Air Pollution and Forest Decline, Volume I, Proc 14th IUFRO-Meeting, Interlaken, 2-8 Oct 1988. Birmensdorf: Eidgenössische Anstalt forstliches Versuchswesen. pp. 209-214.

NEUMANN M, STEMBERGER A (1990) Über Ausmass und Verteilung der Mortalität: Gegenüberstellung von Ergebnissen der Waldzustandsinventur mit früheren Untersuchungen. Cent.bl gesamte Forstwes 107: 63-99.

RENAUD JP, NAGELEISEN LM (2005) Results from the European network for damage monitoring. Bilan de la santé des forêts en 2004. Paris: Département de la santé des forêts. 21 p.

SCHMITT M (1991) Zusammenhang zwischen Blattverlust und Fruktifikation bei Buche. Allg Forst Z Waldwirtsch Umweltvorsorge 10: 501-503.

SEIDLING W (2007) Signals of summer drought in crown condition data from the German Level I network. Eur J Forest Res 126: 529-544.

SOLBERG S ET AL (2009) Analyses of the impact of changes in atmospheric deposition and climate on forest growth in European monitoring plots: A stand growth approach. Forest Ecol Manag 258: 1735-1750. 
SOLBERG S, TVEITE B (2000) Crown density and growth relationships between stands of Picea abies in Norway. Scand J For Res 15: 87-96.

THIMONIER A ET AL (2010) Does exceeding the critical loads for nitrogen alter nitrate leaching, the nutrient status of trees and their crown condition at Swiss Long-term Forest Ecosystem Research (LWF) sites? Eur J For Res 129: 443-461.

THIMONIER A ET AL (2012) Dépôts atmosphériques azotés et leurs effets en forêt: un bilan des sites d'observation à long terme. Schweiz Z Forstwes 163: 343-354. doi: 10.3188/szf.2012.0343 THORNTON PE, RUNNING SW, WHITE MA (1997) Generating surfaces of daily meteorological variables over large regions of complex terrain. J Hydrol 190: 214-251.

ULRICH B, MAYER R, KHANNA PK (1980) Chemical changes due to acid precipitation in a loess-derived soil in central Europe. Soil Sci 130: 193-199.
WALTHERT L, ZIMMERMANN S, BLASER P, LUSTER J, LÜSCHER P (2004) Waldböden der Schweiz. Band 1. Grundlagen und Region Jura. Bern: Hep. 768 p.

WEBSTER R, RIGLING A, WALTHERT L (1996) An analysis of crown condition of Picea, Fagus and Abies in relation to environment in Switzerland. Forestry 69: 347-355.

ZIERL B (2002) Relations between crown condition and ozone and its dependence on environmental factors. Environ Pollut 119: 55-68.

ZIERL B (2004) A simulation study to analyse the relations between crown condition and drought in Switzerland. Forest Ecol Manage 188: 25-38.

ZINGG A (1996) Diameter and basal area increment in permanent growth and yield plots in Switzerland. In: Spiecker $\mathrm{H}$, Mielikäinen K, Köhl M, Skovsgaard JP, editors. Growth trends in European forests. Berlin: Springer. pp. 239-265.

\section{Waldzustand in der Schweiz: Erfassung, Entwicklung und Einflussfaktoren}

Zu Beginn der 1980er-Jahre bestand in Europa die grosse Befürchtung, dass die Luftschadstoffe zu einem verbreiteten Waldsterben oder zumindest zu einer deutlichen Verschlechterung des Waldzustandes führen würden und es zu bedeutenden Produktionsrückgängen kommen könnte. Als wichtigste Merkmale für den Waldzustand wurden in der Schweiz, wie auch in den meisten anderen europäischen Ländern, die Kronenverlichtung der Bäume und die Verfärbung von Blättern und Nadeln aufgenommen. Die Kronenansprachen auf den systematisch verteilten Probeflächen der Sanasilva-Inventur zeigte bis etwa 1995 einen stetigen Anstieg des Anteils der Bäume mit starker Kronenverlichtung. Seither ist kein Trend mehr zu erkennen, wohl jedoch sind grosse Unterschiede zwischen den einzelnen Jahren auszumachen, welche zumeist auf grossflächige Klimaereignisse wie die Stürme Vivian und Lothar und den Hitzesommer 2003 zurückgeführt werden können. Die jährlichen Sterbe- und Nutzungsraten schwanken zwar erheblich, jedoch konnte seit 1985 kein Trend festgestellt werden. Die jährlichen Veränderungen von Kronenverlichtung, Mortalität und Baumwachstum werden vor allem durch klimatische Faktoren bestimmt. Je nach Baumart und Standort wird das Ausmass der Kronenverlichtung neben der Witterung auch durch Nährstoffversorgung, Wasserspeicherfähigkeit des Bodens und zu einem geringen Teil durch Luftverschmutzung erklärt. Obwohl zum Teil ein negativer Einfluss von hohen Stickstoffeinträgen und Ozonbelastungen auf die Belaubung oder Benadelung der Waldbäume belegt wird, haben die Stickstoffeinträge das Baumwachstum auf mit Stickstoff unterversorgten Böden bisher eher erhöht. Der Zustand der Waldbäume in der Schweiz lässt momentan keine unmittelbare Gefährdung durch Luftschadstoffe erkennen. Das Instrument der Erfassung des Kronenzustands allein ist jedoch auch nicht geeignet, den Einfluss der Luftschadstoffe auf den Wald nachzuweisen. Dazu bedarf es genauerer Messungen auf langfristigen Forschungsflächen. Da der Kronenzustand vor allem durch standörtliche Bedingungen und klimatische Ereignisse beeinflusst wird, sollte er in Anbetracht des Klimawandels dennoch weiter erhoben werden.

\section{Etat des forêts suisses: suivi, évolution et facteurs d'influence}

Le début des années 1980 fut marqué en Europe par une vive appréhension: les polluants atmosphériques semblaient pouvoir entraîner à grande échelle la mort des forêts ou du moins une détérioration marquée de leur état, et donc de fortes baisses de productivité. En Suisse, comme dans la plupart des pays européens, la défoliation des arbres et la décoloration de leur feuillage furent choisies comme étant les indicateurs les plus importants de l'état des forêts. Jusqu'en 1995, l'observation de ces deux indicateurs mit tout d'abord en évidence une augmentation constante de la proportion d'arbres présentant une forte défoliation sur les placettes réparties systématiquement dans le cadre de I'inventaire Sanasilva. Aucune tendance n'est perceptible depuis lors, mais certaines années ressortent fortement, principalement en relation avec des événements climatiques de grande ampleur, par exemple les tempêtes Vivian et Lothar ou la canicule de 2003. Les taux de mortalité et d'exploitation fluctuent fortement d'année en année, mais ne présentent aucune tendance depuis 1985. Les différents taux annuels de défoliation, de mortalité et de croissance sont principalement déterminés par les facteurs climatiques. Selon les essences, le taux de défoliation sur un site donné s'explique par les conditions météorologiques ainsi que par la disponibilité en éléments nutritifs, la capacité de rétention en eau et seulement en faible partie par la pollution atmosphérique. Bien que des dépôts élevés d'azote et l'ozone aient un impact en partie négatif sur le taux de défoliation, les mêmes dépôts d'azote ont à ce jour plutôt amélioré la croissance des arbres situés sur des sols forestiers naturellement pauvres en azote. En conclusion, l'état des arbres forestiers n'est pas directement menacé par les polluants atmosphériques. L'observation des houppiers à elle seule n'est toutefois pas un instrument adéquat pour prouver l'impact des polluants atmosphériques sur la forêt. A cet effet, d'autres mesures plus précises sont nécessaires sur des sites de recherche à long terme. Etant donné que l'état des houppiers dépend principalement des conditions stationnelles et des facteurs climatiques, son observation doit toutefois être poursuivie, notamment dans le contexte des changements climatiques. 